\title{
Synthesis, structure, and biological activity of novel heterocyclic sulfonyl-carboximidamides
}

\author{
Katarzyna Gobis • Henryk Foks • Jarosław Sławiński • Artur Sikorski • \\ Damian Trzybiński · Ewa Augustynowicz-Kopeć · Agnieszka Napiórkowska • \\ Krzysztof Bojanowski
}

Received: 23 May 2012/Accepted: 22 November 2012/Published online: 25 January 2013

(C) The Author(s) 2013. This article is published with open access at Springerlink.com

\begin{abstract}
A series of novel heterocyclic sulfonyl-carboximidamides were synthesized in satisfactory yields via condensation of heterocyclic methyl carbimidates with 2-chlorobenzenesulfonamide and 4-chloropyridine-3-sulfonamide. New structures were confirmed by IR and NMR spectra as well as elemental analyses. X-ray crystallography of two derivatives was performed. The single-crystal structures confirmed the presence of a primary amine group in the amidine moiety. All the compounds were screened for their tuberculostatic, antibacterial, and anticancer activities. Preliminary results indicated that target compounds exhibited weak tuberculostatic and antibacterial activities. Seven compounds inhibited the growth of some cancer cell lines, whereas one of the 2-quinoline derivatives displayed favorable activity against all tested cancer cells with $G I_{50}$ values of $0.92-13 \mu \mathrm{M}$.
\end{abstract}

Keywords Sulfonamidine - Heterocycles · Crystal structure - Antimicrobial activity · Antitumor activity $\cdot$ Structure-activity relationship

K. Gobis $(\varangle) \cdot$ H. Foks · J. Sławiński

Department of Organic Chemistry, Medical University

of Gdańsk, Gdańsk, Poland

e-mail: kgobis@gumed.edu.pl

\section{A. Sikorski · D. Trzybiński}

Department of Physical Chemistry, University of Gdańsk,

Gdańsk, Poland

E. Augustynowicz-Kopeć · A. Napiórkowska

Department of Microbiology, Institute of Tuberculosis

and Pulmonary Diseases, Warsaw, Poland

K. Bojanowski

Sunny BioDiscovery, Santa Paula, CA, USA

\section{Introduction}

Sulfonamides are compounds with diverse pharmacological activity. They are known the most for their antibacterial [1] and antihypoglycemic [2] activities. Some of the sulfonamides act as antimycobacterial [3] and antifungal [4] agents. Intensive studies on the antitumor activity of sulfonamides were also carried out [5]. One of the most potent compounds is chloroquinoxaline sulfonamide (CQS) presently in the second phase of clinical trials [6,7]. This compound has a chlorine atom in its structure linked to the quinoxaline ring (C-5) substituted at the $\mathrm{C}-2$ position with a sulfanilamide moiety. The antitumor activity of this sulfonamide is associated with the inhibition of topoisomerase II [8]. Other sulfonamides have also been described as apoptosis promoters $[9,10]$ including sulfonyl-carboximidamides [11].

The amidine functional group is an important structural element of compounds with established pharmacological activity. Amidine derivatives have antidegenerative [12], antitumor [13], and antiplatelet action [14]. They also act as serine protease inhibitors [15] and nitric oxide synthase (NOS) inhibitors [16]. Compounds with anti-HIV [17], antibacterial, and antifungal activities [18] also were found among them. Moreover, the amidine group may be a perfect linker unit that could connect two pharmacophores, e.g., the sulfanilamide moiety and the pyridine or pyrazine system.

These findings prompted us to extend our search for biologically active compounds among nitrogen heterocyclic derivatives. We undertook the synthesis of structures that were condensates of heterocyclic methyl carbimidates with 2-chlorobenzenesulfonamide and 4-chloropyridine3 -sulfonamide. The synthesized compounds were evaluated for their biological activity in vitro: tuberculostatic, 
antibacterial, cytotoxic, and anticancer. We have also determined their crystal structure.

\section{Results and discussion}

The subject of this work was to study the reactions of heterocyclic carbimidates with sulfonamides that have a chlorine atom as a substituent in the ortho position to the sulfonamide group. Carbimidates are compounds of great reactivity. Among others they react with amines giving amidines as the products. A few reactions of alkyl- and phenylcarbimidates with sulfonamides have been also described. As a result, sulfonamidines are formed [19].

One method presented in this article is to use 2-, 3-, and 4-pyridine-, 2-pyrimidine-, 2-pyrazine-, 6-chloro-2-pyrazine-, 6-methoxypyrazine-, and 2-quinolinecarbimidate. An important element of the chosen synthetic route is that there is no need for isolation of carbimidates and they are used in situ after generation from the corresponding carbonitriles in methanol in the presence of DBU (1,8-diazabicycloundec-7-ene). Isolated carbimidates are easy to obtain in pure form and can be also used for 2-pyrazine, 6-chloropyrazine, and 6-methoxypyrazine derivatives. The carbimidates mentioned above underwent reaction with 2-chlorobenzenesulfonamide and 4-chloropyridine-3-sulfonamide. The reactions were carried out in a methanol solution of DBU. That led to the formation of amidine structures with the 2-chlorobenzenesulfonyl or 4-chloropyridine-3-sulfonyl substituent (1-12, 15, 16; Scheme 1). The amidine structures $\mathbf{9}, \mathbf{1 0}, \mathbf{1 3}$, and 14 were prepared from pure isolated carbimidates by refluxing equimolar amounts of the reagents in diglyme (bis(2-methoxyethyl) ether).

In the ${ }^{1} \mathrm{H}$ NMR spectra of the target amidines the signals for all the protons of aromatic systems were observed and two signals for the NH groups were shifted from each other at about $1 \mathrm{ppm}$. These separated signals could be due to the

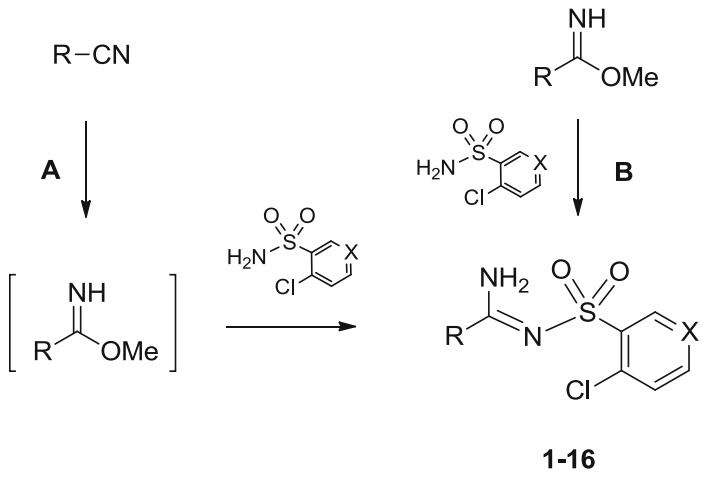

Scheme 1 amino-imine structure adopted by the obtained derivatives (Fig. 1, structure B), as suggested by Northey and coworkers [20]. They could also be the result of the magnetic inequivalence of $\mathrm{NH}$ protons in the amine moiety upon formation of a hydrogen bond in the case of heterocyclic compounds in which the amidine group is in the $\alpha$ position to the nitrogen atom of the heterocyclic ring (structure A), as shown in the previous article [21].

Such a structure could also be stabilized by hydrogen bonding between the second proton of the amine group and the oxygen atom of the sulfonyl moiety. This issue was resolved by X-ray studies performed on derivatives $\mathbf{3}$ and 4. The obtained results revealed the tautomeric structure A (Fig. 2a, b). Thus, formation of hydrogen bonds is the reason for the magnetic inequivalence of the protons of the amino group and separate signals in the ${ }^{1} \mathrm{H}$ NMR spectra of the synthesized compounds.

\section{Crystal structure of compounds $\mathbf{3}$ and $\mathbf{4}$}

In the molecules of the title compounds (Fig. 2a, b) the bond lengths and angles characterizing the geometry of the pyridine skeleton, amino and sulfonyl groups, and benzene ring are typical for this group of compounds [21], although earlier reports from other authors suggested the existence of imine tautomeric structures. Comparison of molecules of $\mathbf{3}$ and $\mathbf{4}$ shows that the structures of both compounds are very similar (Table 1 ).

However, we can observe differences in the dihedral angles and molecular interactions in the crystal packing. With respective average deviations from planarity of $0.006(1)$ and $0.007(3) \AA$, the nearly planar pyridine ring and aromatic fragment (2-chlorobenzene in compound $\mathbf{3}$ and 2-chloro-3pyridine in compound 4) are oriented at an angle of 60.6(2) and $69.1(3)^{\circ}$ to each other, for I and II, respectively. In the packing of both compounds, the amino group participates in the intramolecular $\mathrm{N}-\mathrm{H} \cdots \mathrm{O}$ and intermolecular $\mathrm{N}-\mathrm{H} \cdots \mathrm{O}$ interactions (Fig. 2c, d). This group is also engaged in $\mathrm{N}-\mathrm{H} \cdots \mathrm{N}$ intermolecular interactions, where the acceptor of the $\mathrm{H}$ atom is the endocyclic $\mathrm{N}$ atom from the pyridine (compound $\mathbf{3}$ ) or chloropyridine (compound 4) rings. The neighboring molecules in $\mathbf{3}$ and $\mathbf{4}$ are also linked through the $\mathrm{C}-\mathrm{H} \cdots \mathrm{Cl}$ hydrogen bond. Additionally, weak $\mathrm{C}-\mathrm{H} \cdots \mathrm{O}$ hydrogen bonds are observed in the crystal packing of $\mathbf{4}$.

\section{Biological activity}

Four of the obtained sulfonyl-carboximidamides $(\mathbf{2}, \mathbf{7}, \mathbf{8}$, 10) were evaluated for their in vitro tuberculostatic activity against the Mycobacterium tuberculosis $\mathrm{H}_{37} \mathrm{Rv}$ strain and two "wild" strains isolated from tuberculosis patients: one (sp. 210) resistant to $p$-aminosalicylic acid (PAS), isonicotinic acid hydrazide (INH), ethambutol (ETB), and 
Fig. 1 Possible tautomeric structures of sulfonylcarboximidamides<smiles>O=S(=O)(N=C1N[N]C2=C1CCCC2)c1ccccc1</smiles>

A<smiles>C=Cc1ncnc(C2=NCCCC2)n1</smiles>

B

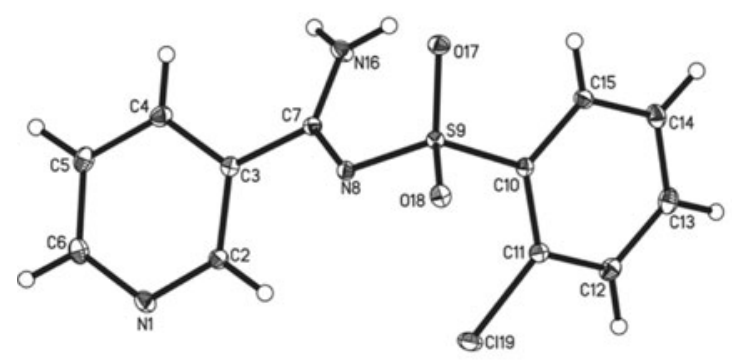

A

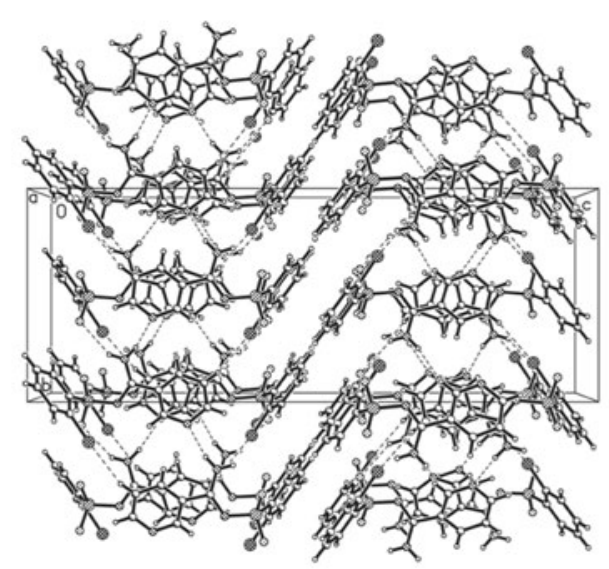

C

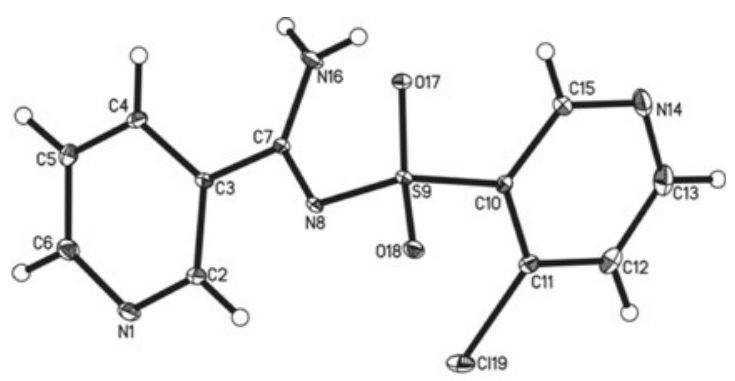

B

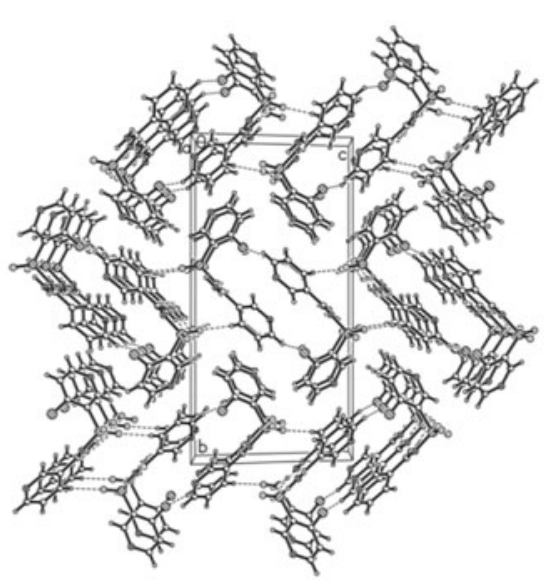

D

Fig. 2 Structure of $\mathbf{3}$ (a) and $\mathbf{4}$ (b) showing $25 \%$ probability displacements for ellipsoid. $\mathrm{H}$ atoms shown as small spheres of arbitrary radius. The arrangement of the molecules in the crystal

rifampicin (RFP), and the another (sp. 192) fully sensitive to the administrated tuberculostatics. Isoniazid (INH) was used as a reference drug. The tested compounds showed rather weak tuberculostatic activity, weaker than the reference INH (MIC $0.5-1.0 \mu \mathrm{g} / \mathrm{cm}^{3}$ ). For all the compounds the determined MIC values were $25-50 \mu \mathrm{g} / \mathrm{cm}^{3}$ against the three tested strains (Table 2).

The compounds were also tested for their antibacterial activity against $P$. acnes (ATCC 11827) and Brevibacterium linens (ATCC 9174). All of the synthesized sulfonylcarboximidamides (1-13) exhibited activity with MICs greater than $256 \mu \mathrm{g} / \mathrm{cm}^{3}$, which meant that those values did not fit standard test concentrations. All synthesized compounds were tested on $B$. linens but no test compound had a MIC of less than $100 \mu \mathrm{g} / \mathrm{cm}^{3}$. The compounds were then structure of $\mathbf{3}$ (c) and $\mathbf{4}$ (d) viewed along $b$ axis. Dashed lines $\mathrm{N}-\mathrm{H} \cdots \mathrm{O}, \mathrm{N}-\mathrm{H} \cdots \mathrm{N}, \mathrm{C}-\mathrm{H} \cdots \mathrm{Cl}$, and $\mathrm{C}-\mathrm{H} \cdots \mathrm{O}$ interactions

tested on P. acnes. Only three of the tested compounds (1, 2, 15) exhibited moderate antibacterial activity with MIC values of $12.5-50 \mu \mathrm{g} / \mathrm{cm}^{3}$. For all other compounds the MIC values were above $100 \mu \mathrm{g} / \mathrm{cm}^{3}$. Compound 15 was the most active (MIC $12.5 \mu \mathrm{g} / \mathrm{cm}^{3}$ ).

The most antibacterially potent sulfonylcarboximidamides $\mathbf{1}, \mathbf{2}$, and $\mathbf{1 5}$ were then tested for their effects on the proliferation of neonatal human dermal fibroblasts (ATCC PCS-201-010). MAP (magnesium ascorbyl phosphate) and bFGF (basic fibroblast growth factor) were used as the positive control (Fig. 3). Compound 2 had no cytotoxic activity. Irrespective to the compound concentration the cell growth remained at the level corresponding to the watertreated control. Compound $\mathbf{1}$ had a weak inhibitory activity. For low compound concentrations $\left(6.25-12.5 \mu \mathrm{g} / \mathrm{cm}^{3}\right)$ no 
Table 1 Crystal data and structure refinement for compounds 3 and $\mathbf{4}$

\begin{tabular}{|c|c|c|}
\hline & Compound $\mathbf{3}$ & Compound 4 \\
\hline Empirical formula & $\mathrm{C}_{12} \mathrm{H}_{10} \mathrm{ClN}_{3} \mathrm{O}_{2} \mathrm{~S}$ & $\mathrm{C}_{11} \mathrm{H}_{9} \mathrm{ClN}_{4} \mathrm{O}_{2} \mathrm{~S}$ \\
\hline Formula weight & 295.74 & 296.73 \\
\hline Temperature/K & $295(2)$ & $295(2)$ \\
\hline Wavelength/A & 1.54184 & 1.54184 \\
\hline Crystal system & Orthorhombic & Monoclinic \\
\hline Space group & Pbca & $P 2_{1} / n$ \\
\hline \multicolumn{3}{|l|}{ Unit cell dimensions } \\
\hline$a / \AA$ & $7.7435(2)$ & $5.5337(5)$ \\
\hline$b / \AA$ & $10.9172(2)$ & $20.8163(9)$ \\
\hline$c / \AA$ & $29.3315(6)$ & $10.6688(6)$ \\
\hline$\beta /^{\circ}$ & & $104.298(8)$ \\
\hline$V / \AA^{3}$ & $2,479.61(9)$ & $1,190.88(14)$ \\
\hline$Z$ & 8 & 4 \\
\hline$D_{\text {calcd }} / \mathrm{mg} \mathrm{m}^{-3}$ & 1.584 & 1.655 \\
\hline Absorption coefficient $/ \mathrm{mm}^{-1}$ & 4.330 & 4.536 \\
\hline$F(000)$ & 1,216 & 608 \\
\hline Crystal size $/ \mathrm{mm}^{3}$ & $0.40 \times 0.12 \times 0.10$ & $0.34 \times 0.18 \times 0.13$ \\
\hline$\Theta$ range for data collection/deg & $3.01-67.18$ & $4.25-67.35$ \\
\hline Limiting indices & $\begin{array}{l}-7 \leq h \leq 8,-13 \leq k \leq 13 \\
-34 \leq l \leq 34\end{array}$ & $\begin{array}{l}-5 \leq h \leq 6,-24 \leq k \leq 24 \\
-12 \leq l \leq 12\end{array}$ \\
\hline Reflections collected/unique & $7,923 / 2,179\left[R_{\mathrm{int}}=0.0581\right]$ & $5,600 / 20,929\left[R_{\mathrm{int}}=0.0961\right]$ \\
\hline Completeness $2 \Theta=50.00 \%$ & 98.2 & 97.6 \\
\hline Refinement method & Full-matrix least-squares on $F^{2}$ & \\
\hline Data/restraints/parameters & $2,179 / 0 / 172$ & $2,092 / 0 / 178$ \\
\hline Goodness-of-fit on $F^{2}$ & 1.079 & 1.058 \\
\hline Final $R$ indices $[I>2 \sigma(I)]$ & $\begin{array}{l}R_{1}=0.0523 \\
w R_{2}=0.1316\end{array}$ & $\begin{array}{l}R_{1}=0.0645 \\
w R_{2}=0.1567\end{array}$ \\
\hline$R$ indices (all data) & $\begin{array}{l}R_{1}=0.0555 \\
w R_{2}=0.1352\end{array}$ & $\begin{array}{l}R_{1}=0.1037 \\
w R_{2}=0.1811\end{array}$ \\
\hline Largest diff. peak and hole/e $\AA^{-3}$ & 0.313 and -0.494 & 0.352 and -0.580 \\
\hline
\end{tabular}

cytotoxic effect was evident. In the range $25-100 \mu \mathrm{g} / \mathrm{cm}^{3}$ a linear relation of the cytotoxic effect was observed with an $88 \%$ growth inhibitory activity at a concentration of $100 \mu \mathrm{g} / \mathrm{cm}^{3}$. Compound $\mathbf{1 5}$ was strongly cytotoxic, even at the lowest concentration tested $\left(6.25 \mu \mathrm{g} / \mathrm{cm}^{3}-70 \%\right.$ growth inhibitory activity).

In view of the cytotoxic activity of compound $\mathbf{1 5}$, it was of interest to determine whether those compounds had an antitumor potential. Compounds 2-16 were tested in the framework of the Development Therapeutic Program (DTP) at the National Cancer Institute (Bethesda, MD, USA) on a panel of 60 human tumor cell lines derived from nine different cancer types: leukemia, lung, colon, CNS, melanoma, ovarian, renal, prostate, and breast. Among the compounds 2-16 tested in the preliminary NCI-60 onedose screen test seven of them (44\%) exhibited distinct growth inhibition $(\Delta \mathrm{GI})$ properties (Table 3$)$. Three compounds $(\mathbf{2}, \mathbf{7}, \mathbf{1 1})$ were active towards one renal cancer UO-31 cell line. These compounds inhibited the growth of that cell line with $\Delta$ GI of from 19.4 to $21.1 \%$. Derivatives 4, 10, and 12 exhibited activity against two cell lines. Compound $\mathbf{4}$ was potent towards the melanoma MALME$3 \mathrm{M}$ cell line ( $\Delta$ GI $20.0 \%$ ) and the renal cancer A498 cell line $(\Delta \mathrm{GI} 24.1 \%)$. Compound $\mathbf{1 0}$ exhibited activity against CNS cancer SNB-75 cell line ( $\Delta$ GI $19.3 \%$ ) and compound 11 against melanoma MALME-3M cell line ( $\Delta$ GI $22.6 \%$ ). Derivative 12 was active towards two cell lines, non-small cell lung cancer HOP-92 ( $\Delta$ GI $25.9 \%)$ and NCI-H522, against which the compound exhibited cytotoxic activity ( $\Delta$ GI $142.9 \%$ ). Derivative 15 was the most potent compound. That compound exhibited activity towards eleven cell lines: leukemia HL-60 ( $\Delta$ GI $19.7 \%)$ and K-562 ( $\Delta$ GI $35.9 \%$ ), non-small cell lung cancer NCI-H460 ( $\Delta$ GI $19.6 \%)$, colon cancer HT29 ( $\Delta$ GI $60.4 \%)$, KM12 ( $\Delta$ GI $23.0 \%$ ) and SW-620 ( $\Delta$ GI $24.7 \%)$, melanoma MDA-MB$435(\Delta$ GI $80.4 \%$ ), ovarian cancer NCI/ADR RES ( $\Delta$ GI $42.9 \%)$, renal cancer TK-10 ( $\Delta$ GI $21.7 \%)$, breast cancer MCF7 ( $\Delta$ GI $15.7 \%$ ) and MDA-MB-468 ( $\Delta$ GI $42.2 \%)$. 
Table 2 Antibacterial activity of the synthesized compounds against $P$. acnes and M. tuberculosis

\author{
Minimum inhibitory \\ concentrations for $P$. acnes were \\ assessed optically as the lowest \\ concentration of a test material \\ which caused no bacteria \\ growth. MICs for $M$. \\ tuberculosis strains were \\ determined by twofold serial \\ dilution method for \\ microdilution plates and for \\ mycobacterial strains by \\ twofold classical test-tube \\ method of successive dilution \\ INH isoniazid, - not tested

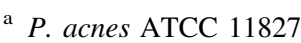

\begin{tabular}{|c|c|c|c|c|}
\hline \multirow[t]{3}{*}{ No. } & \multicolumn{4}{|c|}{$\mathrm{MIC} / \mu \mathrm{g} / \mathrm{cm}^{3}$} \\
\hline & \multirow[t]{2}{*}{ P. acnes ${ }^{\mathrm{a}}$} & \multicolumn{3}{|c|}{ M. tuberculosis } \\
\hline & & $\mathrm{H}_{37} \mathrm{Rv}$ & sp. 192 & sp. 210 \\
\hline 1 & 25 & - & - & - \\
\hline 2 & 50 & 50 & 50 & 50 \\
\hline 3 & $>100$ & - & - & - \\
\hline 4 & $>100$ & - & - & - \\
\hline 5 & $>100$ & - & - & - \\
\hline 6 & $>100$ & - & - & - \\
\hline 7 & $>100$ & 50 & 50 & 50 \\
\hline 8 & $>100$ & 50 & 50 & 50 \\
\hline 9 & $>100$ & - & - & - \\
\hline 10 & $>100$ & 50 & 25 & 50 \\
\hline 11 & $>100$ & - & - & - \\
\hline 12 & $>100$ & - & - & - \\
\hline 13 & $>100$ & - & - & - \\
\hline 14 & $>100$ & - & - & - \\
\hline 15 & 12.5 & - & - & - \\
\hline 16 & $>100$ & - & - & - \\
\hline INH & - & 0.5 & 0.5 & 1.1 \\
\hline
\end{tabular}

Compound 15 was selected for further studies in five concentrations in the range of $10^{-4}$ to $10^{-8} \mathrm{M}$. The activity of the compound was expressed by three dose-response parameters: $G_{50}$ - the molar concentration that inhibits $50 \%$ net cell growth, TGI-the molar concentration leading to total growth inhibition, $L C_{50}$-molar concentration leading to $50 \%$ net cell death (Table 4). Derivative $\mathbf{1 5}$ was the most potent towards the leukemia SR cell line $\left(G I_{50}\right.$ $0.92 \mu \mathrm{M})$, the non-small cell lung cancer NCI-H522 $\left(G I_{50}\right.$ $2.28 \mu \mathrm{M})$, the CNS cancer SNB-75 cell line $\left(G I_{50} 2.52 \mu \mathrm{M}\right)$, the melanoma MDA-MB-435 cell line $\left(G I_{50} 1.13 \mu \mathrm{M}\right)$, the ovarian cancer NCI/ADR-RES cell line $\left(G I_{50} 2.32 \mu \mathrm{M}\right)$, and the breast cancer MDA-MB-468 cell line $\left(G I_{50} 2.01 \mu \mathrm{M}\right)$. In general, compound $\mathbf{1 5}$ exhibited the highest activity against leukemia cell lines. The $G I_{50}$ mean value for that cell line panel was $3.52 \mu \mathrm{M}$.

\section{Conclusion}

A series of novel heterocyclic sulfonyl-carboximidamides with different nitrogen heterocyclic systems were synthesized successfully via condensation of heterocyclic methyl carbimidates with 2-chlorobenzenesulfonamide and 4-chloropyridine-3-sulfonamide. Structures of all these new compounds were confirmed by IR and NMR spectra as well as elemental analyses. X-ray crystallography of compounds 3 and 4 demonstrated the presence of the amine tautomeric structure. The antimicrobial activities of the synthesized compounds were evaluated against $P$. acnes and B. linens as well as M. tuberculosis. The results showed that the synthesized sulfonamide derivatives exhibited rather poor antimicrobial activities in vitro. Seven compounds $(\mathbf{2}, \mathbf{4}, \mathbf{7}$, 10-12, 15) were able to inhibit the growth of some cancer cell lines, whereas the 2-quinoline derivative $\mathbf{1 5}$ showed the highest activity with $G I_{50}$ values of from 0.92 to $13.00 \mu \mathrm{M}$.

\section{Experimental}

All materials and solvents were of analytical reagent grade. Thin-layer chromatography was performed on Merck silica gel $60 \mathrm{~F}_{254}$ plates and visualized with UV. The results of elemental analyses $(\% \mathrm{C}, \mathrm{H}, \mathrm{N})$ for all obtained compounds were in agreement with calculated values within $\pm 0.3 \%$. ${ }^{1} \mathrm{H}$ NMR spectra in DMSO- $d_{6}$ were recorded on Varian Unity Plus (500 MHz) and Varian Gemini (200 MHz) instruments (Varian, Palo Alto, CA). IR spectra were determined as $\mathrm{KBr}$ pellets of the solids on a Satellite FT-IR spectrophotometer (Mattson Instruments, Madison, WI). Electrospray MS analyses for compounds 1, 6, 9, 14, and 16 were performed on an HCT Ultra Bruker Daltonics spectrometer operating in positive- and negative-ion modes (sheath gas $\mathrm{N}_{2}$, temperature $300{ }^{\circ} \mathrm{C}$, flow $7 \mathrm{dm}^{3} / \mathrm{min}$, pressure $10 \mathrm{psi}(689.48 \mathrm{hPa})$; capillary voltage in positive ion mode $+4 \mathrm{kV}$, in negative ion mode $-4 \mathrm{kV}$ ). 


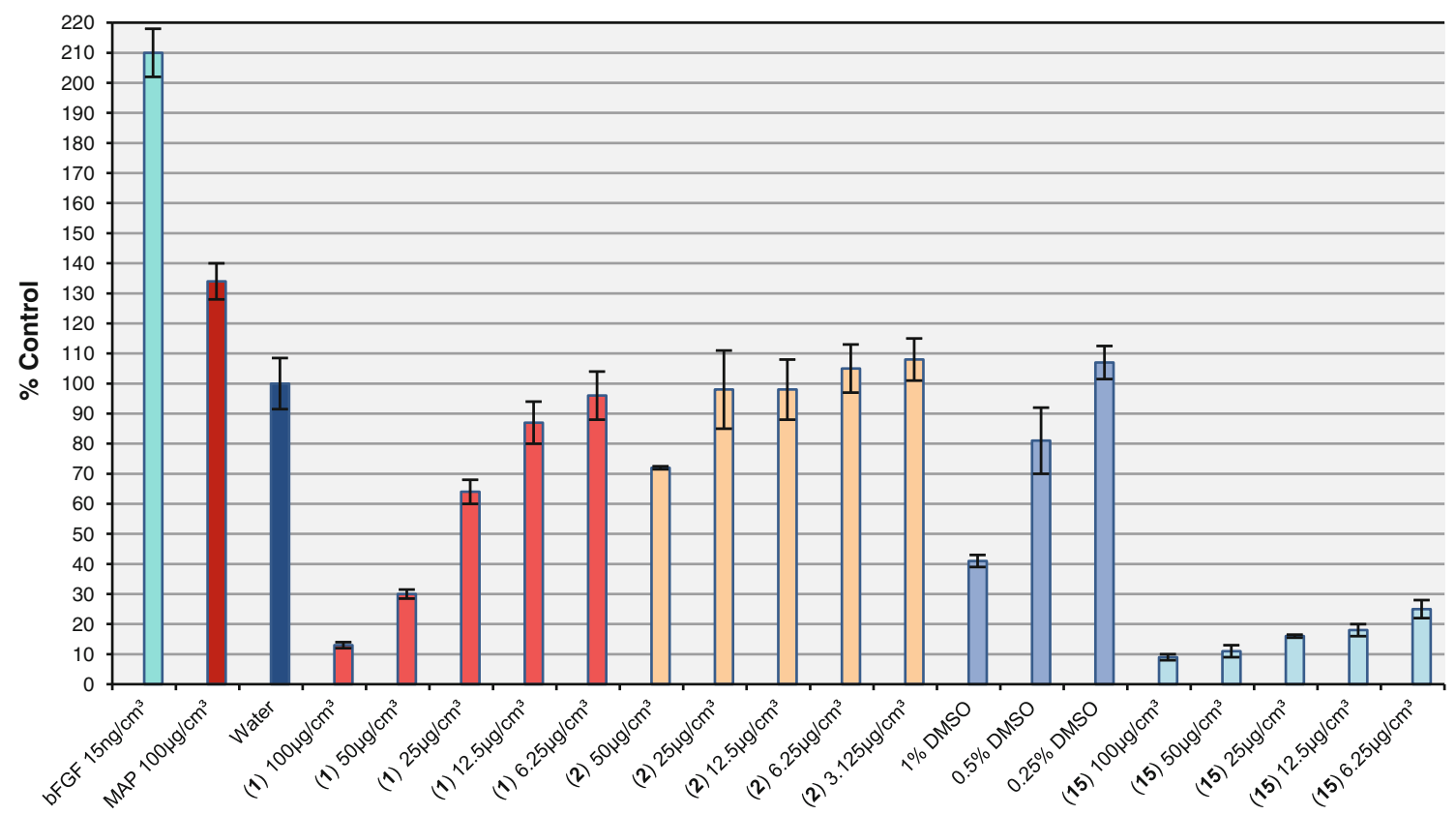

Fig. 3 Total cell/protein count for compounds 1, 2, and 15

Table 3 One-dose screening data of in vitro tumor growth inhibition for compounds $\mathbf{2}, \mathbf{4}$, $7,10-12$, and 15 at a dose of $10 \mu \mathrm{M}$
Data obtained from the NCI-60

DTP human tumor cell line screening

${ }^{\text {a }}$ MIG_MID mean graph midpoint, i.e., arithmetical mean value of growth percent for all tested cell lines

${ }^{\mathrm{b}}$ Cytotoxic effect (lethality)

\begin{tabular}{|c|c|c|c|c|}
\hline No. & $\begin{array}{l}\text { Growth percent } \\
\text { mean values } \\
\left(\mathrm{MG}_{\text {_MID }}\right)^{\mathrm{a}} / \%\end{array}$ & Panel & Cell line & $\begin{array}{l}\text { Growth } \\
\text { inhibition } \\
\Delta \mathrm{GI} / \%\end{array}$ \\
\hline 2 & 98.2 & Renal cancer & UO-31 & 20.4 \\
\hline \multirow[t]{2}{*}{4} & 104.8 & Melanoma & MALME-3 M & 20.0 \\
\hline & & Renal cancer & A498 & 24.1 \\
\hline 7 & 103.0 & Renal cancer & UO-31 & 19.4 \\
\hline 10 & 101.9 & CNS cancer & SNB-75 & 19.3 \\
\hline 11 & 102.1 & Renal cancer & UO-31 & 22.6 \\
\hline \multirow[t]{2}{*}{12} & 97.8 & Non-small cell lung cancer & HOP-92 & 25.9 \\
\hline & & & NCI-H522 & $142.9^{\mathrm{b}}$ \\
\hline \multirow[t]{11}{*}{15} & 53.4 & Leukemia & HL-60 & 19.7 \\
\hline & & & K-562 & 35.9 \\
\hline & & Non-small cell lung cancer & NCI-H460 & 19.6 \\
\hline & & Colon cancer & HT29 & $60.4^{\mathrm{b}}$ \\
\hline & & & KM12 & 23.0 \\
\hline & & & SW-620 & 24.7 \\
\hline & & Melanoma & MDA-MB-435 & $80.4^{\mathrm{b}}$ \\
\hline & & Ovarian cancer & NCI/ADR-RES & 42.9 \\
\hline & & Renal cancer & TK-10 & 21.7 \\
\hline & & Breast cancer & MCF7 & 15.7 \\
\hline & & & MDA-MB-468 & 42.2 \\
\hline
\end{tabular}

Compound samples were prepared in acetonitrile $(\mathbf{1}, \mathbf{9}, \mathbf{1 4}$, 16) or methanol (6). Melting points were determined with a Boethius apparatus (Franz Küstner Nachf. KG, Dresden, Germany). Methyl pyrazine-2-carbimidate and methyl 6-methoxypyrazine-2-carbimidate required for syntheses of compounds 9,10 and 13,14 were obtained according to the method described earlier by Foks and co-workers [22, 23]. Reaction yield and compound characteristics were found to be identical with those described (m.p. 115-116 and $100-101{ }^{\circ} \mathrm{C}$, respectively). 
Table 4 Inhibition of in vitro human cancer cell lines growth by compound 15

\begin{tabular}{|c|c|c|}
\hline Panel/cell line & $G I_{50}^{\mathrm{a}} / \mu \mathrm{M}$ & $\mathrm{TGI}^{\mathrm{b}} / \mu \mathrm{M}$ \\
\hline \multicolumn{3}{|l|}{ Leukemia } \\
\hline CCRF-CEM & 3.65 & c \\
\hline HL-60 (TB) & 2.29 & 8.41 \\
\hline K-562 & 3.11 & c \\
\hline MOLT-4 & 5.91 & $\mathrm{c}$ \\
\hline RPMI-8226 & 5.27 & c \\
\hline SR & 0.92 & 21.40 \\
\hline \multicolumn{3}{|c|}{ Non-small cell lung cancer } \\
\hline A549/ATCC & 5.37 & $\mathrm{c}$ \\
\hline EKVX & 8.75 & c \\
\hline HOP-62 & 5.37 & c \\
\hline HOP-92 & 9.34 & 90.80 \\
\hline NCI-H226 & 10.80 & c \\
\hline NCI-H23 & 5.44 & c \\
\hline NCI-H322M & 5.19 & c \\
\hline NCI-H460 & 4.02 & c \\
\hline NCI-H522 & 2.28 & 7.62 \\
\hline \multicolumn{3}{|l|}{ Colon cancer } \\
\hline COLO 205 & 3.68 & 16.00 \\
\hline HCC-2998 & 9.63 & 59.40 \\
\hline НCT-116 & 4.66 & $\mathrm{c}$ \\
\hline HCT-15 & 4.23 & $\mathrm{c}$ \\
\hline HT29 & 3.40 & 14.90 \\
\hline KM12 & 3.29 & c \\
\hline SW-620 & 3.45 & c \\
\hline \multicolumn{3}{|l|}{ CNS cancer } \\
\hline SF-268 & 6.79 & c \\
\hline SF-295 & 3.83 & 86.30 \\
\hline SF-539 & 4.19 & c \\
\hline SNB-19 & 5.61 & c \\
\hline SNB-75 & 2.52 & 30.60 \\
\hline U251 & 4.48 & c \\
\hline \multicolumn{3}{|l|}{ Melanoma } \\
\hline LOX IMVI & 4.05 & c \\
\hline MALME-3M & 5.42 & c \\
\hline M-14 & 3.61 & c \\
\hline MDA-MB-435 & 1.13 & 3.78 \\
\hline SK-MEL-2 & 3.40 & c \\
\hline SK-MEL-28 & 5.45 & 12.00 \\
\hline SK-MEL-5 & 3.31 & 50.50 \\
\hline UACC-257 & 11.70 & c \\
\hline UACC-62 & 6.07 & c \\
\hline \multicolumn{3}{|l|}{ Ovarian cancer } \\
\hline IGROV1 & 9.42 & c \\
\hline OVCAR-3 & 9.27 & 3.78 \\
\hline OVCAR-4 & 9.15 & 9.53 \\
\hline OVCAR-5 & 7.71 & c \\
\hline OVCAR-8 & 5.74 & 12.00 \\
\hline
\end{tabular}

Table 4 continued

\begin{tabular}{|c|c|c|}
\hline Panel/cell line & $G I_{50}^{\mathrm{a}} / \mu \mathrm{M}$ & $\mathrm{TGI}^{\mathrm{b}} / \mu \mathrm{M}$ \\
\hline NCI/ADR-RES & 2.32 & 50.50 \\
\hline SK-OV-3 & 3.93 & $\mathrm{c}$ \\
\hline \multicolumn{3}{|l|}{ Renal cancer } \\
\hline $786-0$ & 4.94 & c \\
\hline A498 & 8.08 & c \\
\hline ACHN & 7.19 & 13.10 \\
\hline CAKI-1 & 5.21 & c \\
\hline RXF 393 & 4.02 & 35.90 \\
\hline $\mathrm{SN} 12 \mathrm{C}$ & 7.67 & c \\
\hline TK-10 & 6.02 & 7.08 \\
\hline UO-31 & 7.26 & 48.50 \\
\hline \multicolumn{3}{|l|}{ Prostate cancer } \\
\hline PC-3 & 9.59 & c \\
\hline DU-145 & 4.96 & c \\
\hline \multicolumn{3}{|l|}{ Breast cancer } \\
\hline MCF-7 & 3.28 & c \\
\hline MDA-MB-231/ATCC & 13.00 & c \\
\hline HS 578T & 8.98 & c \\
\hline BT-549 & 5.08 & 56.00 \\
\hline $\mathrm{T}-47 \mathrm{D}$ & 7.04 & c \\
\hline MDA-MB-468 & 2.01 & 6.19 \\
\hline
\end{tabular}

Data obtained from the NCI-60 DTP human tumor cell line screening Omitted $L C_{50}$ values (molar concentration leading to $50 \%$ net cell death) were $>100 \mu \mathrm{M}$ except SK-MEL-5 cell line in melanoma panel $(36.2 \mu \mathrm{M})$

a $\mathrm{GI}_{50}$ the molar concentration that inhibits $50 \%$ net cell growth

b TGI the molar concentration leading to total growth inhibition

${ }^{c}$ Determined TGI values were $>100 \mu \mathrm{M}$

General method A for the synthesis of sulfonylcarboximidamides 1-12, 15, 16

The respective carbonitrile $(5 \mathrm{mmol})$ was dissolved in $10 \mathrm{~cm}^{3}$ of methanol and $0.1 \mathrm{~cm}^{3}(0.7 \mathrm{mmol})$ of DBU was added. The mixture was refluxed for $0.5 \mathrm{~h}$ required for methyl carbimidate formation. Then 2-chlorobenzenesulfonamide or 4-chloropyridine-3-sulfonamide (4 mmol) was added. The mixture was refluxed for another $3 \mathrm{~h}$. The solvent was evaporated under vacuum and $30 \mathrm{~g}$ of ice was added. The precipitate was filtered and recrystallized from a suitable solvent.

\section{Alternative method $B$ for the synthesis of sulfonyl- carboximidamides $9,10,13,14$}

Methyl pyrazine-2-carbimidate or methyl 6-methoxypyrazine2-carbimidate $(3 \mathrm{mmol})$ and the respective sulfonamide ( $3 \mathrm{mmol}$ ) were refluxed in $5 \mathrm{~cm}^{3}$ of diglyme for $15 \mathrm{~min}$. Then the mixture was cooled and $30 \mathrm{~g}$ of ice was added. The 
precipitate was filtered and recrystallized from a suitable solvent with addition of activated carbon.

$N^{\prime}$-[(2-Chlorophenyl)sulfonyl]-2-pyridinecarboximidamide $\left(\mathbf{1}, \mathrm{C}_{12} \mathrm{H}_{10} \mathrm{ClN}_{3} \mathrm{O}_{2} \mathrm{~S}\right)$

The crude product was recrystallized from methanol affording $1.1 \mathrm{~g}(93 \%)$ 1. M.p.: $140-142{ }^{\circ} \mathrm{C}$; IR (KBr): $\bar{v}=3,423,3,211(v \mathrm{~N}-\mathrm{H}), 1,613(v \mathrm{C}=\mathrm{N}), 1,583(v \mathrm{C}=\mathrm{C})$, 1,537 ( $v \mathrm{~N}-\mathrm{H}), 1,292,1,284,1,157\left(v \mathrm{SO}_{2}\right), 1,041(\delta \mathrm{C}-\mathrm{H})$, $828,759(\gamma \mathrm{C}-\mathrm{H}), 587(\gamma \mathrm{N}-\mathrm{H}) \mathrm{cm}^{-1}$; ${ }^{1} \mathrm{H}$ NMR $(200 \mathrm{MHz})$ : $\delta=7.51-7.70$ (m, 4H, $2 \mathrm{H} \mathrm{Ph}$ and $2 \mathrm{H}$ pyridine), 7.95-8.18 (m, $3 \mathrm{H}, 2 \mathrm{H} \mathrm{Ph}$ and $1 \mathrm{H}$ pyridine), 8.40 (br s, $1 \mathrm{H}, \mathrm{NH}+\mathrm{D}_{2} \mathrm{O}$ exchangeable), 8.70 (d, $J=4.7 \mathrm{~Hz}, 1 \mathrm{H}$, pyridine), 9.18 (br s, $1 \mathrm{H}, \mathrm{NH}+\mathrm{D}_{2} \mathrm{O}$ exchangeable) $\mathrm{ppm} ;{ }^{13} \mathrm{C} \mathrm{NMR}$ $(50 \mathrm{MHz}): \delta=123.26,127.75,127.92,129.60,131.56$, 131.60, 134.13, 138.46, 139.63, 148.69, 149.28, 159.76 ppm; $\operatorname{MS}(-): m / z=611\left(100 \%,[2 \mathrm{M}+\mathrm{Na}-3 \mathrm{H}]^{-}\right), 294(32 \%$, $\left.[\mathrm{M}-2 \mathrm{H}]^{-}\right), 258\left(28 \%,[\mathrm{M}-2 \mathrm{H}-\mathrm{Cl}]^{-}\right) ; \mathrm{MS}(+): \mathrm{m} / \mathrm{z}=613$ $\left(25 \%,[2 \mathrm{M}+\mathrm{Na}-\mathrm{H}]^{+}\right), 318\left(100 \%,[\mathrm{M}+\mathrm{Na}-\mathrm{H}]^{+}\right), 298$ $\left(71 \%,[\mathrm{M}+2 \mathrm{H}]^{+}\right)$.

$N^{\prime}$-[(4-Chloropyridin-3-yl)sulfonyl]-2-pyridine-

carboximidamide $\left(2, \mathrm{C}_{11} \mathrm{H}_{9} \mathrm{ClN}_{4} \mathrm{O}_{2} \mathrm{~S}\right)$

This compound was recrystallized from ethanol affording 0.82 g (69 \%) 2. M.p.: $150-152{ }^{\circ} \mathrm{C}$; IR (KBr): $\bar{v}=3,430$, 3,390, 3,323 ( $v \mathrm{~N}-\mathrm{H}), 1,629(v \mathrm{C}=\mathrm{N}), 1,587$ ( $\vee \mathrm{C}=\mathrm{C})$, $1,559(v \mathrm{~N}-\mathrm{H}), 1,297,1,275,1,151\left(v_{\mathrm{SO}}\right), 1,115,833(\delta$ $\mathrm{C}-\mathrm{H}), 592(\gamma \mathrm{N}-\mathrm{H}) \mathrm{cm}^{-1} ;{ }^{1} \mathrm{H}$ NMR $(200 \mathrm{MHz}): \delta=7.67$ $(\mathrm{t}, J=5.9 \mathrm{~Hz}, 1 \mathrm{H}$, pyridine $), 7.96-8.13(\mathrm{~m}, 2 \mathrm{H}, 1 \mathrm{H}$ pyridine and $1 \mathrm{H}$ 4-chloropyridine), 8.57 (br s, $1 \mathrm{H}$, $\mathrm{NH}+\mathrm{D}_{2} \mathrm{O}$ exchangeable), 8.70-8.77 (m, 2H, $1 \mathrm{H}$ pyridine and $1 \mathrm{H}$ 4-chloropyridine), 9.22 ( $\mathrm{s}, 1 \mathrm{H}, 4$-chloropyridine), 9.32 (br s, $1 \mathrm{H}, \mathrm{NH}+\mathrm{D}_{2} \mathrm{O}$ exchangeable) ppm; ${ }^{13} \mathrm{C} \mathrm{NMR}$ (50 MHz): $\delta=123.41,126.75,128.04,135.75,138.50$, $141.92,148.50$ (2C), 149.38, 154.45, 160.03 ppm.

(Z)-N'-[(2-Chlorophenyl)sulfonyl]-3-pyridine-

carboximidamide $\left(3, \mathrm{C}_{12} \mathrm{H}_{10} \mathrm{ClN}_{4} \mathrm{O}_{2} \mathrm{~S}_{2}\right)$

This compound was recrystallized from dioxane affording 0.51 g (43 \%) 3. M.p.: $188-190{ }^{\circ} \mathrm{C}$; IR (KBr): $\bar{v}=3,341$ ( $v \mathrm{~N}-\mathrm{H}), 3,156(v \mathrm{C}-\mathrm{H}), 1,644(v \mathrm{C}=\mathrm{N}), 1,591 \quad(v \mathrm{C}=\mathrm{C})$, $1,546(\delta \mathrm{N}-\mathrm{H}), 1,295,1,281,1,162,1,146\left(v \mathrm{SO}_{2}\right), 1,042(\delta$ $\mathrm{C}-\mathrm{H}), 839,761(\gamma \mathrm{C}-\mathrm{H}), 591(\gamma \mathrm{N}-\mathrm{H}) \mathrm{cm}^{-1} ;{ }^{1} \mathrm{H} \mathrm{NMR}$ $(200 \mathrm{MHz}): \delta=7.49-7.65(\mathrm{~m}, 4 \mathrm{H}, 2 \mathrm{H} \mathrm{Ph}$ and $2 \mathrm{H}$ pyridine), $8.12-8.21$ (m, 2H, $\mathrm{Ph}), 8.50$ (br s, 1H, $\mathrm{NH}+\mathrm{D}_{2} \mathrm{O}$ exchangeable $), 8.74(\mathrm{~d}, J=4.4 \mathrm{~Hz}, 2 \mathrm{H}$, pyridine), 9.00 (s, $1 \mathrm{H}$, pyridine), 9.39 (br s, $1 \mathrm{H}, \mathrm{NH}+\mathrm{D}_{2} \mathrm{O}$ exchangeable) $\mathrm{ppm} ;{ }^{13} \mathrm{C}$ NMR $(50 \mathrm{MHz}): \delta=123.76$, 127.65 (2C), 129.59, 129.71, 131.49, 131.97, 133.99, 136.04, 139.81, 149.03, 153.09, 161.94 ppm.

(Z)-N'-[(4-Chloropyridin-3-yl)sulfonyl]-3-pyridinecarboximidamide $\left(4, \mathrm{C}_{11} \mathrm{H}_{9} \mathrm{ClN}_{4} \mathrm{O}_{2} \mathrm{~S}\right)$

This compound was recrystallized from dioxane affording 0.36 g (30 \%) 4. M.p.: $192-194{ }^{\circ} \mathrm{C}$; IR (KBr): $\bar{v}=3,439$,
3,330 ( $v \mathrm{~N}-\mathrm{H}), 1,620(v \mathrm{C}=\mathrm{N}), 1,554,1,514(v \mathrm{C}=\mathrm{C}), 1,283$, $1,146\left(v_{\mathrm{SO}_{2}}\right), 836,795(\gamma \mathrm{C}-\mathrm{H}), 592(\gamma \mathrm{N}-\mathrm{H}) \mathrm{cm}^{-1} ;{ }^{1} \mathrm{H}$ NMR (200 MHz): $\delta=7.49-7.56(\mathrm{~m}, 1 \mathrm{H}$, pyridine), 7.78 (d, $J=5.4 \mathrm{~Hz}, 1 \mathrm{H}$, 4-chloropyridine), 8.17-8.19 (m, 1H, pyridine), 8.22 (br s, $1 \mathrm{H}, \mathrm{NH}+\mathrm{D}_{2} \mathrm{O}$ exchangeable), 8.74 $8.77(\mathrm{~m}, 2 \mathrm{H}, 1 \mathrm{H}$ pyridine and $1 \mathrm{H}$ 4-chloropyridine), 9.00 (d, $J=2.4 \mathrm{~Hz}, 1 \mathrm{H}$, pyridine), 9.20 (s, 1H, 4-chloropyridine), 9.48 (br s, $1 \mathrm{H}, \mathrm{NH}+\mathrm{D}_{2} \mathrm{O}$ exchangeable) ppm; ${ }^{13} \mathrm{C} \mathrm{NMR}$ $(50 \mathrm{MHz}): \delta=124.08,126.78,129.60,135.75$ (2C), $141.93,148.58,149.38,152.60,154.39,162.03$ ppm.

$N^{\prime}$-[(2-Chlorophenyl)sulfonyl]-4-pyridinecarboximidamide $\left(\mathbf{5}, \mathrm{C}_{12} \mathrm{H}_{10} \mathrm{ClN}_{3} \mathrm{O}_{2} \mathrm{~S}\right.$ )

This compound was recrystallized from methanol/water (1:1) affording $0.65 \mathrm{~g}(55 \%)$ 5. M.p.: $182-185{ }^{\circ} \mathrm{C}$; IR $(\mathrm{KBr}): \bar{v}=3,387(v \mathrm{~N}-\mathrm{H}), 3,175(v \mathrm{C}-\mathrm{H}), 1,658(v \mathrm{C}=\mathrm{N})$, 1,581 ( $v \mathrm{C}=\mathrm{C}), 1,526(\delta \mathrm{N}-\mathrm{H}), 1,289,1,145\left(v_{\mathrm{SO}}\right), 850$, $749(\gamma \mathrm{C}-\mathrm{H}), 591(\gamma \mathrm{N}-\mathrm{H}) \mathrm{cm}^{-1} ;{ }^{1} \mathrm{H}$ NMR $(500 \mathrm{MHz})$ : $\delta=7.56(\mathrm{t}, J=7.8 \mathrm{~Hz}, 1 \mathrm{H}, \mathrm{Ph}), 7.65(\mathrm{~m}, 2 \mathrm{H}, \mathrm{Ph}), 7.75$ (d, $J=5.4 \mathrm{~Hz}, 1 \mathrm{H}$, pyridine), $8.14(\mathrm{~d}, J=7.3 \mathrm{~Hz}, 1 \mathrm{H}$, $\mathrm{Ph}$ ), 8.56 (br s, $1 \mathrm{H}, \mathrm{NH}+\mathrm{D}_{2} \mathrm{O}$ exchangeable), 8.74 (d, $J=5.9 \mathrm{~Hz}, 1 \mathrm{H}$, pyridine), 9.42 (br s, $1 \mathrm{H}, \mathrm{NH}+\mathrm{D}_{2} \mathrm{O}$ exchangeable) $\mathrm{ppm} ;{ }^{13} \mathrm{C}$ NMR $(50 \mathrm{MHz}): \delta=121.92$ (2C), 127.70, 129.56, 131.53, 131.99, 134.11, 139.61, 141.12, 150.59 (2C), 161.79 ppm.

$N^{\prime}$-[(4-Chloropyridin-3-yl)sulfonyl]-4-pyridine-

carboximidamide $\left(6, \mathrm{C}_{11} \mathrm{H}_{9} \mathrm{ClN}_{4} \mathrm{O}_{2} \mathrm{~S}\right)$

This compound was recrystallized from dioxane affording $0.71 \mathrm{~g}(60 \%)$ 6. M.p.: $215-218{ }^{\circ} \mathrm{C}$; IR (KBr): $\bar{v}=3,385$, 3,330 ( $v$ N-H), 3,088, 2,964 ( $v$ C-H), 1,660 ( $v \mathrm{C}=\mathrm{N}), 1,570$ $(v \mathrm{C}=\mathrm{C}), 1,533(\delta \mathrm{N}-\mathrm{H}), 1,280,1,116\left(\nu \mathrm{SO}_{2}\right), 854,769(\gamma$ $\mathrm{C}-\mathrm{H}), 594(\gamma \mathrm{N}-\mathrm{H}) \mathrm{cm}^{-1}$; ${ }^{1} \mathrm{H}$ NMR $(200 \mathrm{MHz}): \delta=7.76$ $7.80(\mathrm{~m}, 3 \mathrm{H}, 2 \mathrm{H}$ pyridine and $1 \mathrm{H}$ 4-chloropyridine), $8.72-$ $8.77 \mathrm{~m}, 4 \mathrm{H}, 2 \mathrm{H}$ pyridine and $1 \mathrm{H} 4$-chloropyridine and $1 \mathrm{H}$ $\mathrm{NH}+\mathrm{D}_{2} \mathrm{O}$ exchangeable), 9.19 (s, 1H, 4-chloropyridine), 9.55 (br s, $1 \mathrm{H}, \mathrm{NH}+\mathrm{D}_{2} \mathrm{O}$ exchangeable) ppm; ${ }^{13} \mathrm{C} \mathrm{NMR}$ $(50 \mathrm{MHz}): \delta=122.52$ (2C), 126.53, 135.64, 141.84, 141.95, 149.34 (2C), 149. 67, 154.48, 161.86 ppm; MS $(-): m / z=297\left(26 \%,[\mathrm{M}]^{-}\right), 295\left(66 \%,[\mathrm{M}-2 \mathrm{H}]^{-}\right), 259$ $\left(100 \%,[\mathrm{M}-2 \mathrm{H}-\mathrm{Cl}]^{-}\right) ; \mathrm{MS}(+): m / z=297\left(70 \%,[\mathrm{M}]^{+}\right)$, $153\left(100 \%,\left[\mathrm{M}-\mathrm{H}-\mathrm{Cl}-\mathrm{C}_{5} \mathrm{H}_{3} \mathrm{~N}-\mathrm{NH}_{2}-\mathrm{O}\right]^{+}\right)$.

$N^{\prime}$-[(2-Chlorophenyl)sulfonyl]pyrimidine-2-

carboximidamide $\left(7, \mathrm{C}_{11} \mathrm{H}_{9} \mathrm{ClN}_{4} \mathrm{O}_{2} \mathrm{~S}\right)$

This compound was recrystallized from methanol affording $0.99 \mathrm{~g}(83 \%)$ 7. M.p.: $191-192{ }^{\circ} \mathrm{C}$; IR (KBr): $\bar{v}=3,396$, 3,309 ( $v$ N-H), 1,623 ( $v \mathrm{C}=\mathrm{N}), 1,554,1,391$ ( $v \mathrm{C}=\mathrm{C})$, 1,279, 1,151 ( $\left.v \mathrm{SO}_{2}\right), 838,693(\gamma \mathrm{C}-\mathrm{H}), 589(\gamma \mathrm{N}-\mathrm{H})$ $\mathrm{cm}^{-1} ;{ }^{1} \mathrm{H}$ NMR $(200 \mathrm{MHz}): \delta=7.51-7.65(\mathrm{~m}, 3 \mathrm{H}, \mathrm{Ph})$, $7.70(\mathrm{t}, J=5.0 \mathrm{~Hz}, 1 \mathrm{H}$, pyrimidine), $8.13(\mathrm{~d}, J=7.2 \mathrm{~Hz}$, $2 \mathrm{H}, \mathrm{Ph}$ ), 8.54 (br s, $1 \mathrm{H}, \mathrm{NH}+\mathrm{D}_{2} \mathrm{O}$ exchangable), 9.00 (d, $J=5.0 \mathrm{~Hz}, 2 \mathrm{H}$, pyrimidine), 9.15 (br s, $1 \mathrm{H}, \mathrm{NH}+$ $\mathrm{D}_{2} \mathrm{O}$ exchangable) ppm; ${ }^{13} \mathrm{C}$ NMR (50 MHz): $\delta=123.73$, 
$127.73,129.49,131.66,131.89,134.08,139.76,158.20$ (2C), 158.89, $159.83 \mathrm{ppm}$.

$N^{\prime}$-[(4-Chloropyridin-3-yl)sulfonyl]pyrimidine-2carboximidamide $\left(\mathbf{8}, \mathrm{C}_{10} \mathrm{H}_{8} \mathrm{ClN}_{5} \mathrm{O}_{2} \mathrm{~S}\right)$

This compound was recrystallized from dioxane affording 0.62 g $(52 \%)$ 8. M.p.: $162-163{ }^{\circ} \mathrm{C}$; IR $(\mathrm{KBr}): \bar{v}=3,288$ $(\vee \mathrm{N}-\mathrm{H}), 1,623(\vee \mathrm{C}=\mathrm{N}), 1,560,1,397(\vee \mathrm{C}=\mathrm{C}), 1,291$, 1,219, 1,147 $\left(v \mathrm{SO}_{2}\right), 840(\gamma \mathrm{C}-\mathrm{H}), 600(\gamma \mathrm{N}-\mathrm{H}) \mathrm{cm}^{-1} ;{ }^{1} \mathrm{H}$ NMR (200 MHz): $\delta=7.69-7.77(\mathrm{~m}, 2 \mathrm{H}, 1 \mathrm{H}$ pyrimidine and $1 \mathrm{H}$ 4-chloropyridine $), 8.75(\mathrm{~d}, J=4.3 \mathrm{~Hz}, 1 \mathrm{H}$, 4-chloropyridine), 8.85 (br s, $1 \mathrm{H}, \mathrm{NH}+\mathrm{D}_{2} \mathrm{O}$ exchangable), $8.97(\mathrm{~d}, J=4.5 \mathrm{~Hz}, 2 \mathrm{H}$, pyrimidine), $9.14(\mathrm{~s}, 1 \mathrm{H}$, 4-chloropyridine), 9.39 (br s, $1 \mathrm{H}, \mathrm{NH}+\mathrm{D}_{2} \mathrm{O}$ exchangeable) ppm; ${ }^{13} \mathrm{C}$ NMR (50 MHz): $\delta=123.73,126.67,136.07$, $141.94,149.25,154.26,158.37$ (2C), 160.53, 161.25 ppm.

$N^{\prime}$-[(2-Chlorophenyl)sulfonyl]pyrazine-2-

carboximidamide $\left(\mathbf{9}, \mathrm{C}_{11} \mathrm{H}_{9} \mathrm{ClN}_{4} \mathrm{O}_{2} \mathrm{~S}\right)$

This compound was recrystallized from ethanol affording $0.55 \mathrm{~g}(46 \%) 9$ for method $\mathrm{A}$ and $0.68 \mathrm{~g}(76 \%)$ for method B. M.p.: $173-174{ }^{\circ} \mathrm{C}$; IR (KBr): $\bar{v}=3,438,3,356$, 3,329, 3,254 ( $\vee \mathrm{N}-\mathrm{H}), 3,092(\vee \mathrm{C}-\mathrm{H}), 1,615(\vee \mathrm{C}=\mathrm{N})$, $1,337,1,278,1,182,1,153\left(v \mathrm{SO}_{2}\right), 1,041(\delta \mathrm{C}-\mathrm{H}), 592(\gamma$ $\mathrm{N}-\mathrm{H}) \mathrm{cm}^{-1} ;{ }^{1} \mathrm{H}$ NMR $(500 \mathrm{MHz}): \delta=7.57-7.59(\mathrm{~m}, 1 \mathrm{H}$, $\mathrm{Ph})$, 7.66-7.67 (m, 2H, Ph), $8.18(\mathrm{~d}, J=7.3 \mathrm{~Hz}, 1 \mathrm{H}, \mathrm{Ph})$, 8.53 (br s, $1 \mathrm{H}, \quad \mathrm{NH}+\mathrm{D}_{2} \mathrm{O}$ exchangeable), $8.20(\mathrm{t}$, $J=2.4 \mathrm{~Hz}, 1 \mathrm{H}$, pyrazine), $8.81(\mathrm{~d}, J=2.4 \mathrm{~Hz}, 1 \mathrm{H}$, pyrazine), 9.20 (d, $J=1.4 \mathrm{~Hz}, 1 \mathrm{H}$, pyrazine), 9.34 (br s, $1 \mathrm{H}, \mathrm{NH}+\mathrm{D}_{2} \mathrm{O}$ exchangable) ppm; ${ }^{13} \mathrm{C}$ NMR $(50 \mathrm{MHz})$ : $\delta=127.76, \quad 129.69,131.58,132.03,134.22,139.42$, $144.03,144.28$ (2C), $148.58,158.79$ ppm; MS (-): $\mathrm{m} / \mathrm{z}=613\left(92 \%,[2 \mathrm{M}+\mathrm{Na}-3 \mathrm{H}]^{-}\right), 297\left(43 \%,[\mathrm{M}]^{-}\right)$, $295\left(100 \%,[\mathrm{M}-2 \mathrm{H}]^{-}\right), 259\left(79 \%,[\mathrm{M}-2 \mathrm{H}-\mathrm{Cl}]^{-}\right) ; \mathrm{MS}$ $(+): m / z=615\left(29 \%,[2 \mathrm{M}+\mathrm{Na}-\mathrm{H}]^{+}\right), 319(100 \%$, $\left.[\mathrm{M}+\mathrm{Na}-\mathrm{H}]^{+}\right), 153\left(71 \%,\left[\mathrm{M}-\mathrm{Cl}-\mathrm{C}_{6} \mathrm{H}_{4}-\mathrm{NH}_{2}-\mathrm{O}\right]^{+}\right)$.

$N^{\prime}$-[(4-Chloropyridin-3-yl)sulfonyl]pyrazine-2-

carboximidamide $\left(\mathbf{1 0}, \mathrm{C}_{10} \mathrm{H}_{8} \mathrm{ClN}_{5} \mathrm{O}_{2} \mathrm{~S}\right)$

This compound was recrystallized from ethanol affording $0.79 \mathrm{~g}(66 \%) \mathbf{1 0}$ for method $\mathrm{A}$ and $0.38 \mathrm{~g}(42 \%)$ for method B. M.p.: $187-188{ }^{\circ} \mathrm{C}$; IR (KBr): $\bar{v}=3,380,3,239$ $(v \mathrm{~N}-\mathrm{H}), 1,637(\vee \mathrm{C}=\mathrm{N}), 1,560(\vee \mathrm{C}=\mathrm{C}), 1,547(\delta \mathrm{N}-\mathrm{H})$, $1,285,1,153,1,120\left(v \mathrm{SO}_{2}\right), 853,793(\gamma \mathrm{C}-\mathrm{H}), 600$ $\left(\begin{array}{lllll}\gamma & \mathrm{N}-\mathrm{H}\end{array}\right) \quad \mathrm{cm}^{-1} ; \quad{ }^{1} \mathrm{H} \quad \mathrm{NMR} \quad(200 \mathrm{MHz}): \quad \delta=7.80$ (d, $J=4.8 \mathrm{~Hz}, 1 \mathrm{H}, 4$-chloropyridine), 8.57 (br s, $1 \mathrm{H}$, $\mathrm{NH}+\mathrm{D}_{2} \mathrm{O}$ exchangeable $), 8.77-8.80(\mathrm{~m}, 2 \mathrm{H}, 1 \mathrm{H}$ pyrazine and $1 \mathrm{H} \mathrm{4-chloropyridine),} 8.92(\mathrm{~d}, J=3.2 \mathrm{~Hz}, 1 \mathrm{H}$, 4-chloropyridine), $9.21-9.23$ (m, 2H, pyrazine), 9.43 (br s, $1 \mathrm{H}, \mathrm{NH}+\mathrm{D}_{2} \mathrm{O}$ exchangable) $\mathrm{ppm} ;{ }^{13} \mathrm{C}$ NMR $(50 \mathrm{MHz})$ : $\delta=126.79,135.57,141.98,144.03,144.43$ (2C), 148.68, $149.44,154.53,159.11 \mathrm{ppm}$.
6-Chloro- $N^{\prime}$-(2-chlorophenylsulfonyl)pyrazine-2carboximidamide $\left(\mathbf{1 1}, \mathrm{C}_{11} \mathrm{H}_{8} \mathrm{Cl}_{2} \mathrm{~N}_{5} \mathrm{O}_{2} \mathrm{~S}\right)$

This compound was recrystallized from dioxane/methanol (1:1) affording $1.1 \mathrm{~g}(85 \%)$ 11. M.p.: $195-198{ }^{\circ} \mathrm{C}$; IR $(\mathrm{KBr}): \bar{v}=3,394,3,288,3,236(v \mathrm{~N}-\mathrm{H}), 1,642(v \mathrm{C}=\mathrm{N})$, $1,554(v \mathrm{C}=\mathrm{C}), 1,522(\delta \mathrm{N}-\mathrm{H}), 1,362,1,297,1,152,11,07(\mathrm{v}$ $\left.\mathrm{SO}_{2}\right), 892,790,757(\gamma \mathrm{C}-\mathrm{H}), 567(\gamma \mathrm{N}-\mathrm{H}) \mathrm{cm}^{-1} ;{ }^{1} \mathrm{H}$ NMR (500 MHz): $\delta=7.56-7.59(\mathrm{~m}, 1 \mathrm{H}, \mathrm{Ph}), 7.64-7.69(\mathrm{~m}, 2 \mathrm{H}$, $\mathrm{Ph}), 8.18(\mathrm{~d}, J=7.8 \mathrm{~Hz}, 1 \mathrm{H}, \mathrm{Ph}), 8.56\left(\mathrm{br} \mathrm{s}, 1 \mathrm{H}, \mathrm{NH}+\mathrm{D}_{2} \mathrm{O}\right.$ exchangeable), 9.07 (s, $1 \mathrm{H}$, pyrazine), $9.13(\mathrm{~s}, 1 \mathrm{H}$, pyrazine), 9.34 (br s, $1 \mathrm{H}, \mathrm{NH}+\mathrm{D}_{2} \mathrm{O}$ exchangeable) ppm; ${ }^{13} \mathrm{C} \mathrm{NMR}$ $(50 \mathrm{MHz}): \delta=127.77,129.69,132.04,134.30,139.27$, $142.45,144.56,147.52,148.42,157.80,158.70 \mathrm{ppm}$.

6-Chloro- $N^{\prime}$-[(4-chloropyridin-3-yl)sulfonyl]pyrazine-2carboximidamide $\left(\mathbf{1 2}, \mathrm{C}_{10} \mathrm{H}_{7} \mathrm{Cl}_{2} \mathrm{~N}_{5} \mathrm{O}_{2} \mathrm{~S}\right)$

This compound was recrystallized from methanol/water (1:1) affording $0.64 \mathrm{~g}(48 \%)$ 12. M.p.: $160-162{ }^{\circ} \mathrm{C}$; IR $(\mathrm{KBr}): \bar{v}=3,372(v \mathrm{~N}-\mathrm{H}), 3,092(v \mathrm{C}-\mathrm{H}), 1,656(v \mathrm{~N}=\mathrm{C})$, $1,563,1,547(\vee \mathrm{C}=\mathrm{C}), 1,518(\delta \mathrm{N}-\mathrm{H}), 1,368,1,302,1,150$ $\left(v \mathrm{SO}_{2}\right), 796(\gamma \mathrm{C}-\mathrm{H}), 601(\gamma \mathrm{N}-\mathrm{H}) \mathrm{cm}^{-1} ;{ }^{1} \mathrm{H}$ NMR $(500 \mathrm{MHz}): \delta=7.80(\mathrm{~d}, J=5.4 \mathrm{~Hz}, 1 \mathrm{H}, 4$-chloropyridine), 8.76 (br s, $1 \mathrm{H}, \mathrm{NH}+\mathrm{D}_{2} \mathrm{O}$ exchangeable), 8.78 (d, $J=5.4 \mathrm{~Hz}, 1 \mathrm{H}, 4$-chloropyridine), 9.07 (s, $1 \mathrm{H}$, pyrazine), $9.16(\mathrm{~s}, 1 \mathrm{H}, 4$-chloropyridine), $9.22(\mathrm{~s}, 1 \mathrm{H}$, pyrazine), 9.48 (br s, $1 \mathrm{H}, \mathrm{NH}+\mathrm{D}_{2} \mathrm{O}$ exchangeable) ppm; ${ }^{13} \mathrm{C}$ NMR $(50 \mathrm{MHz}): \delta=126.80,135.48,142.03,142.61,144.45$, $147.49,148.49,149.42,154.55,158.13 \mathrm{ppm}$.

$N^{\prime}$-(2-Chlorophenylsulfonyl)-6-methoxypyrazine-2-

carboximidamide $\left(\mathbf{1 3}, \mathrm{C}_{12} \mathrm{H}_{11} \mathrm{ClN}_{4} \mathrm{O}_{3} \mathrm{~S}\right)$

This compound was recrystallized from methanol affording $0.75 \mathrm{~g}(76 \%)$ 13. M.p.: $227-230{ }^{\circ} \mathrm{C}$; IR (KBr): $\bar{v}=3,401$, 3,248 ( $\vee \mathrm{N}-\mathrm{H}), 1,643(\vee \mathrm{C}=\mathrm{N}), 1,541(\vee \mathrm{C}=\mathrm{C}), 1,381$, $1,320,1,281,1,146,1,106\left(v \mathrm{SO}_{2}\right), 804(\gamma \mathrm{C}-\mathrm{H}), 584,561$ $(\gamma \mathrm{N}-\mathrm{H}) \mathrm{cm}^{-1} ;{ }^{1} \mathrm{H}$ NMR $(200 \mathrm{MHz}): \delta=4.03(\mathrm{~s}, 3 \mathrm{H}$, $\left.\mathrm{OCH}_{3}\right), 7.51-7.65(\mathrm{~m}, 3 \mathrm{H}, \mathrm{Ph}), 8.15-8.19(\mathrm{~m}, 1 \mathrm{H}, \mathrm{Ph}), 8.55$ (s, $1 \mathrm{H}$, pyrazine), $8.73(\mathrm{~s}, 1 \mathrm{H}$, pyrazine), $8.80(\mathrm{br} \mathrm{s}, 1 \mathrm{H}$, $\mathrm{NH}+\mathrm{D}_{2} \mathrm{O}$ exchangeable), 9.00 (br s, $1 \mathrm{H}, \mathrm{NH}+\mathrm{D}_{2} \mathrm{O}$ exchangeable) $\mathrm{ppm} ;{ }^{13} \mathrm{C}$ NMR $(50 \mathrm{MHz}): \delta=54.59$, $127.73,129.61,131.55,132.03,134.16,135.74,139.81$ (2C), 140.87, 158.70, $159.08 \mathrm{ppm}$.

$N^{\prime}$-[(4-Chloropyridin-3-yl)sulfonyl]-6-methoxypyrazine-2carboximidamide $\left(\mathbf{1 4}, \mathrm{C}_{11} \mathrm{H}_{10} \mathrm{ClN}_{5} \mathrm{O}_{3} \mathrm{~S}\right)$

This compound was recrystallized from DMSO affording $0.32 \mathrm{~g}(33 \%)$ 14. M.p.: $265-270{ }^{\circ} \mathrm{C}$ (decomp.); IR (KBr): $\bar{v}=3,337,3,302(v \mathrm{~N}-\mathrm{H}), 3,087(v \mathrm{C}-\mathrm{H}), 1,642(v \mathrm{C}=\mathrm{N})$, $1,547,1,450(\vee \mathrm{C}=\mathrm{C}), 1,383,1,280,1,131\left(v \mathrm{SO}_{2}\right), 1,008$ $(\delta \mathrm{C}-\mathrm{H}), 822,777(\gamma \mathrm{C}-\mathrm{H}), 581(\gamma \mathrm{N}-\mathrm{H}) \mathrm{cm}^{-1} ;{ }^{1} \mathrm{H}$ NMR $(200 \mathrm{MHz}): \delta=4.04\left(\mathrm{~s}, 3 \mathrm{H}, \mathrm{OCH}_{3}\right), 7.08(\mathrm{~d}$, $J=5.1 \mathrm{~Hz}, 1 \mathrm{H}, 4$-chloropyridine), 7.55 (s, $1 \mathrm{H}$, pyrazine), 8.75-8.77 (m, 3H, $2 \mathrm{H}$ 4-chloropyridine and $1 \mathrm{H} \mathrm{NH}+\mathrm{D}_{2} \mathrm{O}$ 
exchangeable), $9.22(\mathrm{~s}, 1 \mathrm{H}$, pyrazine), 9.27 (br s, 1H, $\mathrm{NH}+\mathrm{D}_{2} \mathrm{O}$ exchangeable) ppm; ${ }^{13} \mathrm{C}$ NMR $(50 \mathrm{MHz})$ : $\delta=54.64,126.82,135.66,135.88,140.01$ (2C), 140.68, $141.96,149.33,154.49,159.04 \mathrm{ppm}$; MS $(-): \mathrm{m} / z=326$ $\left(15 \%,[\mathrm{M}-2 \mathrm{H}]^{-}\right), 290\left(100 \%,[\mathrm{M}-2 \mathrm{H}-\mathrm{Cl}]^{-}\right)$; $\mathrm{MS}(+)$ : $m / z=341\left(8 \%,\left[\mathrm{M}+2 \mathrm{Na}-\mathrm{H}-\mathrm{NH}_{2}-\mathrm{O}\right]^{+}\right), 153(100 \%$, $\left.\left[\mathrm{M}+\mathrm{H}-\mathrm{Cl}-\mathrm{C}_{5} \mathrm{H}_{3} \mathrm{~N}-\mathrm{NH}_{2}-\mathrm{O}\right]^{+}\right)$.

$N^{\prime}$-(2-Chlorophenylsulfonyl)quinoline-2carboximidamide $\left(\mathbf{1 5}, \mathrm{C}_{16} \mathrm{H}_{12} \mathrm{ClN}_{3} \mathrm{O}_{2} \mathrm{~S}\right)$

This compound was recrystallized from ethanol affording $0.97 \mathrm{~g}(70 \%)$ 15. M.p.: $161-162{ }^{\circ} \mathrm{C}$; IR (KBr): $\bar{v}=3,446$, $3,331(v \mathrm{~N}-\mathrm{H}), 1,640(v \mathrm{C}=\mathrm{N}), 1,616(v \mathrm{C}=\mathrm{C}), 1,527(\delta \mathrm{N}-\mathrm{H})$, $1,277,1,180,1,106\left(v \mathrm{SO}_{2}\right), 1,030(\delta \mathrm{C}-\mathrm{H}), 801,770,628$ $(\gamma \mathrm{C}-\mathrm{H}), 575(\gamma \mathrm{N}-\mathrm{H}) \mathrm{cm}^{-1} ;{ }^{1} \mathrm{H}$ NMR $(200 \mathrm{MHz})$ : $\delta=7.53-7.77(\mathrm{~m}, 4 \mathrm{H}, 2 \mathrm{H} \mathrm{Ph}$ and $2 \mathrm{H}$ quinoline), $7.88(\mathrm{t}$, $J=7.0 \mathrm{~Hz}, 1 \mathrm{H}$, quinoline), $8.06-8.23(\mathrm{~m}, 4 \mathrm{H}, 2 \mathrm{H} \mathrm{Ph}$ and $2 \mathrm{H}$ quinoline), $8.52(\mathrm{~d}, J=8.2 \mathrm{~Hz}, 1 \mathrm{H}$, quinoline), 8.60 (br s, $1 \mathrm{H}, \mathrm{NH}+\mathrm{D}_{2} \mathrm{O}$ exchangeable), 9.30 (br s, $1 \mathrm{H}$, $\mathrm{NH}+\mathrm{D}_{2} \mathrm{O}$ exchangeable) ppm; ${ }^{13} \mathrm{C}$ NMR $(50 \mathrm{MHz})$ : $\delta=119.26, \quad 127.79, \quad 128.34,129.02, \quad 129.39,129.58$, $129.64,131.12,131.64,132.03,134.20,138.52,139.58$, $146.33,148.98,159.57 \mathrm{ppm}$.

$N^{\prime}$-[(4-Chloropyridin-3-yl)sulfonyl]quinoline-2carboximidamide $\left(\mathbf{1 6}, \mathrm{C}_{15} \mathrm{H}_{11} \mathrm{ClN}_{4} \mathrm{O}_{2} \mathrm{~S}\right)$

This compound was recrystallized from dioxane affording $1.1 \mathrm{~g}(80 \%)$ 16. M.p.: $347-350{ }^{\circ} \mathrm{C}$ (decomp.); IR (KBr): $\bar{v}=3,380,3,182(v \mathrm{~N}-\mathrm{H}), 1,637(v \mathrm{C}=\mathrm{N}), 1,560(v \mathrm{C}=\mathrm{C})$, $1,536(\delta \mathrm{N}-\mathrm{H}), 1,300,1,148,1,121\left(v \mathrm{SO}_{2}\right), 813,771,627$ $(\gamma \mathrm{C}-\mathrm{H}), 605,592(\gamma \mathrm{N}-\mathrm{H}) \mathrm{cm}^{-1}$; ${ }^{1} \mathrm{H}$ NMR $(200 \mathrm{MHz})$ : $\delta=7.72-7.81(\mathrm{~m}, 2 \mathrm{H}, 1 \mathrm{H}$ quinoline and $1 \mathrm{H}$ 4-chloropyridine), 7.91 (t, $J=7.0 \mathrm{~Hz}, 1 \mathrm{H}$, quinoline), 8.09-8.19 (m, $3 \mathrm{H}$, quinoline), $8.57(\mathrm{~d}, J=8.8 \mathrm{~Hz}, 1 \mathrm{H}$, quinoline), 8.69 (br s, $1 \mathrm{H}, \mathrm{NH}+\mathrm{D}_{2} \mathrm{O}$ exchangeable), $8.77(\mathrm{~d}, J=5.4 \mathrm{~Hz}$, 1H, 4-chloropyridine), 9.25 (s, 1H, 4-chloropyridine), 9.42 (br s, $1 \mathrm{H}, \mathrm{NH}+\mathrm{D}_{2} \mathrm{O}$ exchangeable) ppm; ${ }^{13} \mathrm{C}$ NMR (50 MHz): $\delta=119.36,126.80,128.38,129.12,129.44$, $129.61,131.19,135.71,138.61,141.98,146.35,148.82$, 149.40, 154.54, $159.90 \mathrm{ppm}$; MS (-): $\mathrm{m} / \mathrm{z}=713(7 \%$, $\left.[2 \mathrm{M}+\mathrm{Na}-3 \mathrm{H}]^{-}\right), 613\left(41 \%,\left[2 \mathrm{M}+\mathrm{Na}-2 \mathrm{Cl}-2 \mathrm{NH}_{2}\right]^{-}\right)$, $283\left(61 \%,\left[\mathrm{M}+\mathrm{Na}-3 \mathrm{H}-\mathrm{Cl}-\mathrm{NH}_{2}-2 \mathrm{O}\right]^{-}\right), 255(100 \%$, $\left.\left[\mathrm{M}+\mathrm{Na}-2 \mathrm{H}-\mathrm{Cl}-\mathrm{C}_{5} \mathrm{H}_{3} \mathrm{~N}\right]^{-}\right) ; \mathrm{MS}(+): \mathrm{m} / \mathrm{z}=715(25 \%$, $\left.[2 \mathrm{M}+\mathrm{Na}-\mathrm{H}]^{+}\right), 353\left(100 \%,\left[\mathrm{M}+\mathrm{Na}-\mathrm{H}-\mathrm{NH}_{2}\right]^{+}\right), 153$ $\left(25 \%,\left[\mathrm{M}-\mathrm{H}-\mathrm{NH}_{2}-\mathrm{Cl}-\mathrm{C}_{5} \mathrm{H}_{3} \mathrm{~N}-\mathrm{SO}_{2}\right]^{+}\right)$.

\section{$X$-ray crystallography}

Good quality single-crystal specimens were selected for the X-ray diffraction experiments at $T=295(2) \mathrm{K}$. They were mounted with epoxy glue at the tip of glass capillaries. Diffraction data were collected on an Oxford Diffraction Gemini R ULTRA Ruby CCD diffractometer with $\mathrm{CuK} \alpha$ radiation $(\lambda=1.54184 \AA)$. The lattice parameters were obtained by least-squares fit to the optimized setting angles of the collected reflections by means of CrysAlis CCD [24]. Data were reduced by using CrysAlis RED [24] software by applying multi-scan absorption corrections (empirical absorption correction using spherical harmonics, implemented in the SCALE3 ABSPACK scaling algorithm). The structural resolution procedure was made using the SHELXS-97 package solving the structures by direct methods and carrying out refinements by full-matrix leastsquares on $F^{2}$ using the SHELXL-97 program [25].

All $\mathrm{H}$ atoms bound with aromatic $\mathrm{C}$ atoms were placed geometrically and refined using a riding model with $\mathrm{C}-\mathrm{H}=0.93 \AA$ and $U_{\text {iso }}(\mathrm{H})=1.2 U_{\text {eq }}(\mathrm{C})$. All $\mathrm{H}$ atoms bound with $\mathrm{N}$ atoms were placed geometrically and refined using a riding model with $\mathrm{N}-\mathrm{H}=0.86 \AA$ and $U_{\text {iso }}(\mathrm{H})=$ $1.2 U_{\mathrm{eq}}(\mathrm{N})$. All interactions demonstrated were found by the PLATON program [26]. The following programs were used to prepare molecular graphics: ORTEPII [27], PLUTO-78 [28], and Mercury [29].

Full crystallographic details for compounds 3 and $\mathbf{4}$, excluding structures features, have been deposited with the Cambridge Crystallographic Data Centre (deposition no. CCDC 869834 \& 869835). These data may be obtained, on request, from the Director, CCDC, 12 Union Road, Cambridge, CB2 1EZ, UK (Tel.: +44-1223-336408; Fax: +441223-336033; E-mail: deposit@ccdc.cam.ac.uk or http:// www.ccdc.cam.ac.uk).

\section{Tuberculostatic activity}

The newly synthesized compounds were examined in vitro for their tuberculostatic activity against $M$. tuberculosis $\mathrm{H}_{37} \mathrm{Rv}$ strain and two "wild" strains isolated from tuberculosis patients: one (sp. 210) resistant to $p$-aminosalicylic acid (PAS), isonicotinic acid hydrazide (INH), etambutol (ETB), and rifampicine (RFP), and the another (sp. 192) fully sensitive to the administered tuberculostatics (Table 2). Investigations were performed by a classical test-tube method of successive dilution in Youmans' modification of the Proskauer and Beck liquid medium containing $10 \%$ of bovine serum [30, 31]. Bacterial suspensions were prepared from 14-day-old cultures of slowly growing strains and from 48-h-old cultures of saprophytic strains $[32,33]$. Solutions of compounds in ethylene glycol were tested. Stock solutions contained $10 \mathrm{mg}$ of compounds in $1 \mathrm{~cm}^{3}$. Dilutions (in geometric progression) were prepared in Youmans' medium. Media containing no investigated substances and containing INH as reference drug were used for comparison. The incubation was performed at a temperature of $37^{\circ} \mathrm{C}$. The MIC values were determined as the minimum concentration inhibiting the growth of the tested tuberculous strains in relation to the probe with no tested compound. 


\section{Antibacterial activity}

Compounds 1-15 were dissolved immediately before use in $100 \%$ DMSO at $5 \mathrm{mg} / \mathrm{cm}^{3}$ and further to $1 \mathrm{mg} / \mathrm{cm}^{3}$ in $10 \%$ DMSO. Compounds were tested at serial dilutions in bacterial broth starting at $100 \mu \mathrm{g} / \mathrm{cm}^{3}$ (final concentration). P. acnes (ATCC 11827, lot 419697) was grown in thioglycollate nutrient broth (Hardy Diagnostics K29) for $72 \mathrm{~h}$ at $33{ }^{\circ} \mathrm{C}$, then inoculated at the density equivalent to 0.5 McFarland standard and incubated with the test materials for another $72 \mathrm{~h}$ in an anaerobic environment. B. linens (ATCC 9174, lot 419862) culture was started from an agar plate, grown in nutrient broth (Hardy Diagnostics $\mathrm{K} 243$ ) for $24 \mathrm{~h}$ at $30{ }^{\circ} \mathrm{C}$, then inoculated at the density equivalent to $1 \mathrm{McF}$ arland standard and incubated with the test materials for another $24 \mathrm{~h}$. At the end of the incubation the MIC was assessed optically as the lowest concentration of a test material which caused no bacteria growth [34]. This optical assessment was further confirmed by measuring the absorbance at $655 \mathrm{~nm}$ with the BioRad 3550-UV microplate reader. The MIC was defined as at least $50 \%$ inhibition of the increase of OD.

\section{Cytotoxic activity}

Passage for normal neonatal human dermal fibroblasts (ATCC PCS-201-010, lot 58243223) were grown in DMEM with $5 \%$ calf serum (Hyclone). For the experiment, cells were plated in DMEM/5\% serum at 2,000 cells/well in 96 well plates (plate 598) and were exposed to test materials for $96 \mathrm{~h}$. MAP and bFGF were used as the positive controls and water, 1, 0.5, and $0.25 \%$ DMSO were used as the negative controls. Plate growth was stopped and cells were stained with a sulforhodamine $\mathrm{B}$ dye [35]. The dye was then dissolved and a colorimetric signal proportional to total cell/protein count was quantified with the BioRad microplate spectrophotometer $3550-\mathrm{UV}$ at $570 \mathrm{~nm}$ with background subtraction at $660 \mathrm{~nm}$ and analyzed with Microplate Manager v.2 software for Macintosh (BioRad). Error bars represent standard errors of the mean (SEM). $P$ values representing statistical significance were calculated using the $t$ test.

\section{Antitumor activity}

Compounds 2-16 were tested in the preliminary screening on a panel of 60 human tumor cell lines in the framework of the in vitro Development Therapeutic Program (DTP) at the National Cancer Institute (Bethesda, MD, USA). Cell lines were derived from nine different cancer types: leukemia, lung, colon, CNS, melanoma, ovarian, renal, prostate, and breast. Compounds were tested at one concentration $(10 \mu \mathrm{M})$. Compound 15, which passed the preliminary screening, was then tested at five different concentrations. Details of the system and the information which is encoded by the activity pattern over all cell lines have been published [36-38]. The antitumor activity of a test compound is given by the parameters for each cell line: $G I_{50}$, i.e., the molar concentration of the compound that inhibits $50 \%$ net cell growth, TGI, i.e., the molar concentration of compound leading to total growth inhibition, and $L C_{50}$, i.e., the molar concentration of the compound leading to $50 \%$ net cell death. Furthermore, a mean graph midpoint (MG_MID) is calculated for each of the mentioned parameters, giving an averaged activity parameter over all cell lines. For the calculation of the MG_MID, insensitive cell lines of the screen are included with the highest concentration tested. The selectivity of a compound with respect to one or more cell lines of the screen is characterized by the high deviation of the particular cell line parameter compared to the MG_MID value.

Acknowledgments The authors are very grateful to Dr. Joel Morris, Chief of Drug Synthesis \& Chemistry Branch, National Cancer Institute (Bethesda, MD) for the in vitro screening.

Open Access This article is distributed under the terms of the Creative Commons Attribution License which permits any use, distribution, and reproduction in any medium, provided the original author(s) and the source are credited.

\section{References}

1. Young LS (2007) Bacteriology, mayor pathogens, and diseases. In: Taylor JB, Triggle DJ (eds) Comprehensive medicinal chemistry II, vol 7. Elsevier Science, Oxford, p 469

2. Shuman CR (1983) Am J Med 75:55

3. Klimešová V, Zahajská L, Waisser K, Kaustová J, Möllmann U (2004) Farmaco 59:279

4. Isik S, Kockar F, Aydin M, Arslan O, Guler OO, Innocenti A, Scozzafava A, Supuran CT (2009) Bioorg Med Chem 17:1158

5. El-Sayed NS, El-Bendary ER, El-Ashy SM, El-Kerdawy MM (2011) Eur J Med Chem 46:3714

6. Rigas JR, Francis PA, Miller VA, Tong WP, Roistacher N, Kris MG, Orazem JP, Young CW, Warrell RP Jr (1995) Cancer Chemother Pharmacol 35:483

7. Bekaii-Saab TS, Mortazavi A, Hicks LG, Zalupski M, Pelley RJ, Chan KK, Kraut EH (2006) Invest New Drugs 24:343

8. Gao H, Yamasaki EF, Chan KK, Shen LL, Snapka RM (2000) Cancer Res 60:5937

9. Ghorab MM, Ragab FA, Hamed MM (2009) Eur J Med Chem 44:4211

10. Kamal A, Dastagiri D, Ramaiah MJ, Reddy JS, Sagar MVR, Reddy TL, Pushpavalli SNCVL, Pal-Bhadra M (2011) Eur J Med Chem 46:5817

11. Elmore SW, Bruncko M, Park C-M (2005) Preparation of $N$ sulfonylcarboximidamides for use as apoptosis promoters. US Patent Appl US 20050272744

12. Panico A, Vicini P, Incert M, Cardile V, Gentile B, Ronsisvalle G (2002) Farmaco 57:671

13. Sienkiewicz P, Bielawski K, Bielawska A, Palka J (2005) Environ Toxicol Pharmacol 20:118 
14. Sielecki TM, Liu J, Mousa SA, Racanelli AL, Hausner EA, Waxler RR, Olson RE (2001) Bioorg Med Chem Lett 11:2201

15. Liebeschuetz JW, Jones SD, Morgan PJ, Marray CW, Rimmer AD, Roscoe JME, Waszkowycz B, Welsh PM, Wylie WA, Young SC, Martin H, Mahler J, Brady L, Wilkinson K (2002) J Med Chem 45:1221

16. Collons JL, Shearer BG, Oplinger JA, Lee S, Garvey EP, Salter M, Dufry C, Burnette TC, Furtine ES (1998) J Med Chem 41:2858

17. Echevarria A, Santos LH, Miller J, Mahmood N (1996) Bioorg Med Chem Lett 6:1901

18. Bedi PMS, Mahajan MP, Kapoor VK (2004) Bioorg Med Chem Lett 14:3821

19. Fujisawa T, Mizuno C (1952) Yakugaku Zasshi 72:694

20. Northey EH, Pierce AE, Kartesz DJ (1942) J Am Chem Soc 64:2760

21. Gobis K, Foks H, Wiśniewska K, Dąbrowska-Szponar M, Augustynowicz-Kopeć E, Napiórkowska A, Sikorski A (2012) Monatsh Chem 143:1161

22. Foks H, Janowiec M (1979) Acta Polon Pharm 36:155

23. Foks H, Manowska W (1976) Pol J Pharmacol Pharm 28:49

24. Oxford Diffraction (2008) CrysAlis CCD and CrysAlis RED. Oxford Diffraction, Abingdon

25. Sheldrick GM (2008) Acta Cryst A 64:112

26. Spek AL (2009) Acta Cryst D 65:148

27. Johnson CK (1976) ORTEP II Report ORNL-5138. Oak Ridge National Laboratory, Oak Ridge
28. Mortherwell S, Clegg S (1978) PLUTO-78. Program for drawing and molecular structure. University of Cambridge, Cambridge, UK

29. Macrae F, Bruno IJ, Chisholm JA, Edgington PR, McCabe P, Pidcock E, Rodriguez-Monge L, Taylor R, van de Streek J, Wood PA (2008) J Appl Cryst 41:466

30. Youmans GP (1947) Am Rev Tuberc 56:376

31. Youmans GP, Youmans AS (1949) J Bacteriol 58:247

32. Atlas RM, Singler JW (1995) Media for clinical microbiology. CRC, Boca Raton

33. Foks H, Buraczewska M, Manowska W, Sawlewicz J (1971) Dissert Pharm Pharmacol 23:49

34. Clinical and Laboratory Standards Institute (2006) Methods for dilution susceptibility tests for bacteria that grow aerobically, 7th edn. Approved Standard M7-A7. CLSI, Wayne

35. Skehan P, Storeng R, Scudiero D, Monks A, McMahon J, Vistica D, Warren JT, Bokesch H, Kenney S, Boyd MR (1990) J Natl Cancer Inst 82:1107

36. Boyd MR (1989) Am Assoc Cancer Res 30:652

37. Monks AP, Scudiero DA, Skehan P, Shoemaker R, Poull KD, Vistica D, Hose C, Langley J, Cronise P, Vaigro-Wolff A (1991) J Natl Cancer Inst 83:757

38. Weinstein JN, Myers TG, Kohn KW, Buolamwini JK, van Osdol WW, Monks AP, Scudeiro DA, Sansville EA, Zaharevitz DW, Bunow RE, Paull KD (1997) Science 275:343 